\title{
Cardiotoxic activity of polymeric 3-alkylpyridinium salts from the marine sponge Reniera sarai Mojca Lunder*1, Lovro Žiberna ${ }^{1}$, Kristina Sepčić ${ }^{2}$ and Gorazd Drevenšek ${ }^{1}$
}

\author{
Address: ${ }^{1}$ Institute of Pharmacology and Experimental Toxicology, Faculty of Medicine, University of Ljubljana, 1000 Ljubljana, Slovenia and \\ ${ }^{2}$ Department of Biology, Biotechnical Faculty, University of Ljubljana, 1000 Ljubljana, Slovenia \\ Email: Mojca Lunder* - mojca.lunder@mf.uni-lj.si \\ * Corresponding author
}

\author{
from 15th Scientific Symposium of the Austrian Pharmacological Society (APHAR) Joint meeting with the Hungarian Society of Experimental and Clinical \\ Pharmacology (MFT) and the Slovenian Pharmacological Society (SDF) \\ Graz, Austria. 19-2I November 2009 \\ Published: 12 November 2009 \\ BMC Pharmacology 2009, 9(Suppl 2):A62 doi:I0.I I86/I47I-22I0-9-S2-A62
}

This abstract is available from: http://www.biomedcentral.com/I47I-22I0/9/S2/A62

(c) 2009 Lunder et al; licensee BioMed Central Ltd.

\section{Background}

Marine sponges are a rich source of novel biologically active substances such as haemolytic proteins, hemagglutinins and proteins with antimicrobial, antiviral and antifungal activity. Toxic water-soluble polymeric 3alkylpyridinum salts (poly-APS) have been isolated from the marine sponge Reniera sarai. Poly-APS has pore-forming properties expressed in a dose-dependent manner. These interactions with cell membranes results in increased $\mathrm{Ca}^{2+}$ permeability. In in vivo experiments performed in rats, poly-APS caused transient bradycardia, prolongation of expirium, lowered blood pressure, formed numerous thrombocyte aggregates and caused death. The aim of the present study was to investigate the direct cardiotoxic effects of poly-APS on isolated rat hearts.

\section{Methods}

Poly-APS isolated from the marine sponge Reniera sarai was purified and lyophilized. The experiments were carried out on the isolated rat hearts (Wistar rats, $\mathrm{n}=30$, weight 250-290 g) of both sexes according to Langendorff. The isolated rat hearts were perfused with different polyAPS concentrations $(1,10$ and $100 \mathrm{nmol} / \mathrm{L}$ and $1 \mu \mathrm{mol} /$ L). During the experiments, the coronary flow rate, lactate dehydrogenase (LDH) release rate, left ventricular pressure, heart rate and duration of arrhythmias were measured.

\section{Results}

The most evident activity in all of the studied parameters was observed in the group perfused with poly-APS in a concentration of $1 \mu \mathrm{mol} / \mathrm{L}$. Only the lowest concentration of poly-APS that was used $(1 \mathrm{nmol} / \mathrm{L})$ did not influence the measured parameters. Poly-APS in a concentration of $1 \mu \mathrm{mol} / \mathrm{L}$ diminished the coronary flow 7.6-fold, increased LDH release rate 12.7 -fold, increased the pressure in left ventricle 2.6-fold and diminished heart rate 2.9-fold compared to the control group (all $\mathrm{p}<0.001$ ). Poly-APS also showed proarrhythmogenic activity.

\section{Conclusion}

Poly-APS salts isolated from the marine sponge Reniera sarai showed potent cardiotoxic activity on isolated rat hearts. 